Department of Medicine, Division of Infectious Diseases, UPMC Health System and the University of Pittsburgh School of Medicine, Pittsburgh, PA 15213, USA

2 Division of Pulmonary and Critical Care Medicine, Department of Medicine, University of Pennsylvania Perelman School of Medicine, Philadelphia, PA, USA

Correspondence to: E K McCreary mccrearye3@upmc.edu Cite this as: BMJ 2021;372:n244 http://dx.doi.org/10.1136/bmj.n244 Published: 27 January 2021

\section{Covid-19 controversies: the tocilizumab chapter}

\author{
The signals are broadly positive, but not definitive \\ Erin K McCreary, ${ }^{1}$ Nuala J Meyer ${ }^{2}$
}

More than two million people have so far died from coronavirus disease 2019 (covid-19) globally. With hospitals and intensive care units at or exceeding capacity in much of the world, discovering life-saving treatments is second only to global vaccination efforts to stop the horrific impact of this disease. Early in the pandemic, elevated inflammatory markers were found to be associated with severe covid-19 and death, leading to exploration of immunomodulatory treatments in clinical trials.

Tocilizumab, a humanized monoclonal antibody that inhibits interleukin 6 mediated signaling by blocking interleukin 6 from binding to receptors, was an early front runner in the race to find treatments for severely ill patients..$^{2-5}$ However, conflicting results from several randomized clinical trials, along with corticosteroids becoming standard care for patients admitted to hospital who required oxygen, tempered enthusiasm for its use. ${ }^{6-9}$ Now, in a linked paper, Veiga and colleagues (doi:10.1136/bmj.n84) report a randomized trial from Brazil that compared tocilizumab with standard care in 129 patients with covid-19. ${ }^{10}$ Surprisingly, the trial was stopped early because tocilizumab was associated with increased deaths at day $15(17 \% v 3 \%$, odds ratio $6.42,95 \%$ confidence interval 1.59 to 43.2 ). So, should tocilizumab be abandoned? The answer is not straightforward.

In recent weeks, preprinted results from 803 critically ill participants in the REMAP-CAP (Randomised, Embedded, Multi-factorial, Adaptive Platform Trial for Community-Acquired Pneumonia) trial found tocilizumab decreased in-hospital mortality compared with standard care $(28 \% v 35.8 \%$, adjusted odds ratio for survival 1.64, 95\% confidence interval 1.14 to 2.35 ) and reduced progression to intubation, extracorporeal membrane oxygenation, or death. ${ }^{11}$ In the REMAP-CAP population, tocilizumab appeared to be life saving.

Possible explanations for these discrepant results might lie in differences in coadministered treatments, populations, or timing. Both REMAP-CAP and the trial by Veiga and colleagues administered corticosteroids to more than $80 \%$ of participants. This is highly relevant given the reported mortality benefit of corticosteroids for patients requiring oxygen, and the potential concern of additive

immunosuppression. ${ }^{12}$ Remdesivir was not used in the trial from Brazil and was used infrequently in REMAP-CAP (about $30 \%$ of participants). Thus, neither steroids nor remdesivir likely explain the difference, although the exact drug, dose, and duration of steroid use in both trials are unknown.

Differences existed in the trial populations, as well as in the timing of treatment with tocilizumab.
Whereas REMAP-CAP enrolled critically ill patients within 24 hours of requirement for high flow oxygen by nasal canula, non-invasive ventilation, or mechanical ventilation, Veiga and colleagues enrolled predominantly moderately ill patients. The deaths in the Brazilian trial were largely among patients who received tocilizumab within 24 hours of mechanical ventilation, suggesting this is not the explanation.

In a third trial (COVACTA (Study to Evaluate the Safety and Efficacy of Tocilizumab in Patients with Severe COVID-19 Pneumonia), also in preprint), a post hoc subgroup analysis of patients requiring high flow oxygen by nasal canula found that tocilizumab significantly improved clinical status at day $14 .{ }^{8} \mathrm{In}$ analyses of the whole trial population, patients given tocilizumab were discharged from hospital earlier ( $20 v 28$ days) and had lower risk of progression to clinical failure than placebo controls, but 28 day mortality did not differ. In COVACTA, participants requiring mechanical ventilation were enrolled a mean of 5 (median 3, range 0-28) days after intubation; perhaps the drug was administered past the "golden hour"? Finally, the EMPACTA (Evaluating Minority Patients with Actemra) placebo controlled trial found that tocilizumab reduced risk of progression to mechanical ventilation or death among patients predominantly receiving low flow oxygen, but did not improve 28 day survival. ${ }^{13}$

Taken together, the randomized evidence published before the REMAP-CAP preprint suggests significantly less clinical deterioration among patients treated with tocilizumab but no mortality benefit across heterogenous populations. ${ }^{14}$

\section{Immune activation}

These trials might have differed in participants' patterns of immune activation. Veiga and colleagues explicitly enriched their trial population by requiring elevated levels of at least two non-specific inflammatory markers. Most participants in REMAP-CAP also met these inflammatory criteria for both $\mathrm{C}$ reactive protein and ferritin. It is, however, unclear whether these non-specific markers are a reasonable sample enrichment strategy for trials of anti-interleukin 6 treatment. Patients admitted to hospital with covid-19 have substantial immune heterogeneity that is not captured by non-specific markers. ${ }^{15}$ Both innate and adaptive immune activation seem to have critical roles, and although a relation might exist between plasma interleukin 6 and dysregulated $\mathrm{T}$ cell activation, this correlation is far from proven. ${ }^{16}$ Even assuming that plasma interleukin 6 is correlated with hyperactive T cell activation, a causal relation is yet to be shown or even inferred between interleukin 6 and unfavorable 
outcomes. Treatments for covid-19 are complex, and controversies remain around selecting the "right" patients, even for treatments now considered standard care.

The signal for harm in the trial by Veiga and colleagues, although alarming, is based on relatively few deaths. The data safety board appropriately prioritized patient safety and recommended stopping the trial early; however, the high death rate in mechanically ventilated patients given tocilizumab and lack of deaths in the controls requiring high flow oxygen or non-invasive ventilation could be due to chance. Effects might be overestimated when trials are stopped early and small datasets present fragile results. ${ }^{17}$

The totality of randomized data evaluating tocilizumab neither overwhelmingly support nor convincingly refute routine use. The harm reported by Veiga and colleagues is an outlier in a small trial. On balance of evidence, tocilizumab is unlikely to be life threatening. The mortality benefit reported by REMAP-CAP is also a statistical outlier, but it is more robust because of the larger population and is consistent with signals of benefit in the sickest patients in COVACTA and EMPACTA. The results of the RECOVERY (Randomised Evaluation of COVID-19 Therapy) trial are eagerly awaited to further inform tocilizumab's role in the management of critically ill patients with covid-19.

Competing interest: The BMJ has judged that there are no disqualifying financial ties to commercial companies. The authors declare the following other interests: EM carries out advisory board work on antibiotics for Shionogi, Summit, Entasis, Merck, and AbbVie and is a site investigator for REMAP-CAP in the US (site did not enroll to interleukin 6ra domain). The BMJ policy on financial interests is here: https://www.bmj.com/sites/default/files/attachments/resources/2016/03/16-current-bmj-educationcoi-form.pdf.

Provenance and peer review: Commissioned; not externally peer reviewed.

1 Zhou F, Yu T, Du R, etal. Clinical course and risk factors for mortality of adult inpatients with COVID-19 in Wuhan, China: a retrospective cohort study. Lancet 2020;395:1054-62. doi: 10.1016/S0140-6736(20)30566-3 pmid: 32171076

2 Oldfield V, Dhillon S, Plosker GL. Tocilizumab: a review of its use in the management of rheumatoid arthritis. Drugs 2009;69:609-32. doi: 10.2165/00003495-200969050-00007 pmid: 19368420

3 Somers EC, Eschenauer GA, Troost JP, etal. Tocilizumab for treatment of mechanically ventilated patients with COVID-19. Clin Infect Dis 2020;ciaa954. doi: 10.1093/cid/ciaa954 pmid: 32651997

4 Xu X, Han M, Li T, etal. Effective treatment of severe COVID-19 patients with tocilizumab. Proc Natl Acad Sci U S A 2020;117:10970-5. doi: 10.1073/pnas.2005615117 pmid: 32350134

5 Si S, Teachey DT. Spotlight on Tocilizumab in the Treatment of CAR-T-Cell-Induced Cytokine Release Syndrome: Clinical Evidence to Date. Ther Clin Risk Manag 2020;16:705-14.pmid: 32801727

6 Stone JH, Frigault MJ, Serling-Boyd NJ, etalBACC Bay Tocilizumab Trial Investigators. Efficacy of Tocilizumab in Patients Hospitalized with Covid-19. N Engl J Med 2020;383:2333-44. doi: 10.1056/NEJMoa2028836 pmid: 33085857

7 Hermine O, Mariette X, Tharaux PL, Resche-Rigon M, Porcher R, Ravaud PCORIMUNO-19 Collaborative Group. Effect of Tocilizumab vs Usual Care in Adults Hospitalized With COVID-19 and Moderate or Severe Pneumonia: A Randomized Clinical Trial. JAMA Intern Med2021;181:32-40. doi: 10.1001/jamainternmed.2020.6820 pmid: 33080017

8 Rosas I, Bräu N, Waters M, etal. Tocilizumab in Hospitalized Patients With COVID-19 Pneumonia.medRxiv .2020:2020.2008.2027.20183442doi: 10.1101/2020.08.27.20183442.

9 Salvarani C, Dolci G, Massari M, etalRCT-TCZ-COVID-19 Study Group. Effect of Tocilizumab vs Standard Care on Clinical Worsening in Patients Hospitalized With COVID-19 Pneumonia: A Randomized Clinical Trial. JAMA Intern Med 2021;181:24-31. doi: 10.1001/jamainternmed.2020.6615 pmid: 33080005

10 Veiga VC, Prats JAGG, Farias DLC, etalCoalition covid-19 Brazil VI Investigators. Effect of tocilizumab on clinical outcomes at 15 days in patients with severe or critical coronavirus disease 2019: randomised controlled trial. BMJ 2021;372:n84. doi: 10.1136/bmj.n84 pmid: 33472855

11 Gordon AC, Mouncey PR, Al-Beidh F, etal. Interleukin-6 Receptor Antagonists in Critically III Patients with Covid-19 - Preliminary report.medRxiv .2021:2021.2001.2007.21249390.

12 Horby P, Lim WS, Emberson JR, etalRECOVERY Collaborative Group. Dexamethasone in Hospitalized Patients with Covid-19 - Preliminary Report. N Engl / Med 2020.pmid: 32678530

13 Salama C, Han J, Yau L, etal. Tocilizumab in Patients Hospitalized with Covid-19 Pneumonia. N Engl J Med 2021;384:20-30. doi: 10.1056/NEJMoa2030340 pmid: 33332779

14 Tocilizumab. Infectious Diseases Society of America Guidelines on the Treatment and Management of Patients with COVID-19. https://www.idsociety.org/practice-guideline/covid-19-guidelinetreatment-and-management/. Accessed 20 Jan 2021.
15 Mathew D, Giles JR, Baxter AE, etalUPenn COVID Processing Unit. Deep immune profiling of COVID-19 patients reveals distinct immunotypes with therapeutic implications. Science 2020;369:eabc8511. doi: 10.1126/science.abc8511 pmid: 32669297

16 Laing AG, Lorenc A, Del Molino Del Barrio I, etal. A dynamic COVID-19 immune signature includes associations with poor prognosis. Nat Med 2020;26:1623-35. doi: 10.1038/s41591-020-1038-6 pmid: 32807934

17 Guyatt GH, Briel M, Glasziou P, Bassler D, Montori VM. Problems of stopping trials early. BMJ 2012;344:e3863. doi: 10.1136/bmj.e3863 pmid: 22705814 